\title{
Research on the Model of Cross-Border Medical Tourism Decision-Making under the Background of Globalization
}

\author{
Xuelan Sun \\ School of Public Administration and Emergency Management, Jinan University, Guangzhou, China \\ Email: sunxuelan2403@gmail.com
}

How to cite this paper: Sun, X.L. (2018) Research on the Model of Cross-Border Medical Tourism Decision-Making under the Background of Globalization. Open Journal of Social Sciences, 6, 230-246. https://doi.org/10.4236/jss.2018.69016

Received: September 4, 2018

Accepted: September 17, 2018

Published: September 20, 2018

Copyright $\odot 2018$ by author and Scientific Research Publishing Inc. This work is licensed under the Creative Commons Attribution International License (CC BY 4.0).

http://creativecommons.org/licenses/by/4.0/

\section{c) (i) Open Access}

\begin{abstract}
With the help of the wave of globalization, the new industry market is constantly emerging, showing a clear trend of "reverse globalization". Among them, cross-border medical tourism and related industries are particularly effective. The flow of cross-border medical tourism patients has changed dramatically. What is the development of international medical tourism industry? Which factors make the "reverse globalization" phenomenon occur on cross-border medical tourism? How do patients decide their medical tourism destination? This paper based on the analysis of relevant literatures and the development of international medical tourism industry, tries to summarize cross-border medical tourism decision-making process, and develops a two-stage model for cross-border medical tourism. Through the analysis of the decision-making process of cross-border medical tourism, this paper considers that a country's economic level, political climate and regulatory policies are important factors influencing people's choice of cross-border medical tourism destinations. We also argue that medical treatment cost, hospital accreditation, quality of care, and physician experience influence patients' choice of medical facility.
\end{abstract}

\section{Keywords}

Globalization, Cross-Border Medical, Medical Tourism, Two-Stage Decision Model

\section{Introduction}

With the development of science and technology, the industrial structure has been continuously upgraded and transformed, and new industries have emerged in the tide of globalization. Medical tourism and related industries have become 
one of the fast-growing and profitable industries in many countries [1]. The medical tourism market has expanded rapidly, and the competition in the cross-border medical tourism market has become increasingly fierce. Thailand, India, Singapore, Jordan, Hungary, South Africa and Mexico have become the main destinations for cross-border medical tourism [2]. In 2000, the total output value of the world medical tourism industry was less than 10 billion US dollars, and in 2004 it reached 40 billion US dollars. In 2006, it increased to 60 billion US dollars. In 2012, it soared to 100 billion US dollars [3], and the development momentum is amazing. According to the latest issue of the International Medical Tourism Association white paper quotes the survey data provided by Stanford Research Institute in 2012, it predicts that by 2017, cross-border medical tourism will bring in $\$ 678.5$ billion in revenue, accounting for $16 \%$ of the world's total tourism revenue. The medical tourism market has broad prospects.

Tourism has a long history, dating back to the early 14th century when European rich people went to Greece to travel to spas, and modern medical tourism began to develop in the late 1990s. The medical tourism industry developed rapidly in the early 21 st century, but so far there is no unified definition [4]. It is generally believed that medical tourism is a new type of tourism service for the purpose of medical, nursing, rehabilitation and tourism for the sake of economic, social and institutional factors [5]. Up to now, the cross-border flow of medical tourism has driven the rapid development of related industries, but it has also experienced a reverse change from the flow of less developed countries to developed countries to the flow of developed countries to new developing countries. In the past 20 years, the phenomenon of "reverse globalization" of medical tourism has become increasingly obvious. People have shown that they have sought medical services from developing countries such as the United States, Canada, the United Kingdom, and Australia to developing countries such as Thailand, India, Malaysia, Hungary, and South Africa [6]. The phenomenon of "reverse globalization" in the flow of cross-border medical tourism tourists is the result of the joint action of "thrust" and "pull" in international trade [7]. Up to now, more than 50 countries in the world have listed medical tourism in the national industrial development of key national development. The "thrust" that led to the surge in cross-border medical tourism and the soaring output value mainly include: high medical expenses, insufficient coverage of medical insurance or limited scope, (such as the United States), waiting for medical treatment for too long (such as Canada, the United Kingdom), backward medical technology (such as Vietnam, Cambodia); "pull" mainly includes: low cost of medical treatment, advanced medical technology, first-class medical services etc. [8]. The proximity of the same geographical system and the consistency of religious beliefs are also important pulls for the development of cross-border medical tourism.

Along with the deepening of globalization reform, the broad development prospects of the medical tourism industry have attracted many countries to compete in the cross-border medical tourism market, and have successively 
shaped their brand advantages with "first world technology, third world price". Asia is one of the most important medical tourism markets, with Thailand, India and Singapore occupying the top three positions [9]. Latin America's Brazil, Costa Rica, Mexico, the Middle East's Jordan, Israel, the United Arab Emirates, Europe's Hungary, Poland, and the Czech Republic will also develop cross-border medical tourism industry as an important economic growth point to play an important role in the international medical tourism arena. The medical tourism market is expanding day by day, and people's choices are becoming more and more abundant, making the decision-making process of cross-border medical tourism more important. Can accurately analyze and grasp the decision-making process and behavioral characteristics of cross-border medical tourism consumers to improve the competition of the domestic medical tourism industry? If a medical tourism destination country wants to take the initiative in fierce competition, acquire more customers, and seize a larger market share, it must fully consider the decision-making process of cross-border medical tourism consumers. With the continuous rise of cross-border medical tourism topics, research and reports on medical tourism at home and abroad have also increased significantly. However, most domestic scholars study the development status, challenges and countermeasures of medical tourism. Most foreign scholars focus on medical tourism case studies. There is relatively little research on the decision-making process and decision-making model of people's cross-border medical tourism. Cross-border medical tourism is a new type of medical industry that is formed by self-issuance at the individual level. The decision-making process of studying cross-border medical tourists has certain significance for industrial development and national strategy. China's medical tourism is still in its infancy, grasping the development status of the medical tourism industry and the decision-making psychology of consumers, which contributes to the strategic positioning and competitive advantage at the national level.

Based on the above analysis, this paper intends to summarize the definitions of medical tourism connotation and types by domestic and foreign scholars, select several representative representatives of medical tourism development, systematically explain the development status and dynamic trends of cross-border medical tourism industry, and combine the cross-border medical tourism industry. The "thrust" and "pull" of development, according to the sequential logic process to sort out the decision-making process of consumers in cross-border medical tourism, and then establish a two-stage decision-making model from the two dimensions of state and medical service, and grasp the cross-border medical tourism. The decision-making behavior and consumption preferences of the crowd are expected to provide reference for the study of medical tourism in China.

\section{Conceptual Framework}

\subsection{The Connotation and Types of Cross-Border Medical Tourism}

In recent years, with the aging of the population, soaring medical costs and 
technological innovation, people's awareness of health care has increased, and projects that enjoy medical services in tourism have gradually been favored. Different from the general tourism industry, the medical tourism industry is a new type of industry combining medical and tourism. It has broken through the traditional mono-structure with the theme of sightseeing and tourism, and has developed into a medical, tourism, rehabilitation and health care system as an integrated industry. Since there is no uniform definition of medical tourism, the International Tourism Organization believes that medical tourism is a new type of tourism service with medical care, disease health and rehabilitation. The definitions of medical tourism in the relevant literatures at home and abroad are also different, but it is generally believed that medical tourism essentially refers to people seeking medical services with higher cost performance due to local medical standards, medical costs, institutional restrictions, and ethical constraints [10].

\subsection{Types and Typical Representatives of Cross-Border Medical Tourism}

Medical tourism is an important branch that cannot be ignored in the field of health care. According to the difference in medical and tourism weights, cross-border medical tourism can be divided into narrow medical tourism and medical tourism with the aim of seeking medical services. According to whether or not surgery is the standard, some scholars divide medical tourism into three categories: general medical examination, invasive surgery and lifestyle medicine [11]. Early cross-border medical tourism provided high-quality medical services to high-income people in developing countries to developed countries. Horowitz and Jones (2007) motivated medical tourists into five categories: price-orientedBecause the cost of medical care in the country is too high to seek low-cost choices abroad; there is no medical insurance-including the uncovered population of medical insurance and the items not covered by medical insurance, resulting in higher costs; dissatisfied with the national medical policy-medical socialization leads to waiting time for medical treatment Long; ethical controversy-some medical services (such as abortion, IVF, stem cell transplant, euthanasia, etc.) that are not yet developed or prohibited by law or that cause ethical controversy abroad; protection of privacy-abroad Perform surgical treatments that require privacy protection (such as degenerative surgery, drug treatment, etc.) [12].

At present, cross-border medical tourism is booming in the international arena. It is a high value-added emerging industry with broad market prospects and has become an important economic growth point in many countries. Thailand, India and Singapore are typical representatives. After the Asian financial turmoil in 1997, the number of private medical institutions in Thailand decreased sharply due to the reduction in the number of medical treatments in the country. In order to revitalize the medical economy, the Thai government began to take measures to develop the medical tourism industry. In 2004, it began to expand 
overseas markets [13]. Thailand's medical beauty, plastic surgery (especially breast enhancement, transsexual) and other special specialist services are prominent. According to the official tourism bureau of Thailand, 600,000 medical tourists from all over the world went to Thailand for medical services in 2004, with an output value of 500 million US dollars. The number of medical tourists in the country reached 1.4 million, making it one of the fastest growing industries in Thailand with a growth rate of $20 \%$ per year. It is expected to complete the annual industry income of US\$6 billion by the end of 2017 [14]. India is a rising star in cross-border medical tourism. It has been known worldwide for cardiovascular medicine, orthopedics and neurosurgery. It combines the advantages of traditional Chinese medicine to introduce Ayurvedic medicine, Siddha medicine, traditional yoga and other special products. India's medical tourists are growing at a rate of $25 \%$ a year, and India's medical tourism market revenue in 2013 reached $\$ 3$ billion [15]. India's world-class medical standards, low medical costs, quality medical team and language advantages have become an important magic weapon for the development of cross-border medical tourism. The World Health Organization ranks Singapore as the best country in the Asian healthcare system, and its sophisticated medical services and high cost performance are important guarantees for the development of high-end medical tourism. Singapore's medical tourism industry revenue reached $\$ 1.5$ billion in 2016 and maintained a growth rate of $13.6 \%$ per year. Compared with the United States, Thailand, India, and Singapore have obvious price advantages in medical tourism service projects (as shown in Table 1).

\section{Analyses}

\subsection{Analysis of Consumer Purchase Decision Process}

Obviously, cross-border medical tourism integrates the medical industry with the tourism industry, involving industries such as hospitals, hotels, restaurants,

Table 1. Comparison of medical project prices between the United States and Asian countries (Unit: US Dollar).

\begin{tabular}{|c|c|c|c|c|c|c|c|}
\hline \multirow{2}{*}{ Medical Project } & \multirow{2}{*}{ US } & \multirow{2}{*}{ India } & \multirow{2}{*}{ Thailand } & \multirow{2}{*}{ Singapore- } & \multicolumn{3}{|c|}{ National Price/US Price } \\
\hline & & & & & India & Thailand & Singapore \\
\hline $\begin{array}{c}\text { Cardiac bypass } \\
\text { surgery }\end{array}$ & 130,000 & 10,000 & 11,000 & 18,500 & $8 \%$ & $8 \%$ & $14 \%$ \\
\hline $\begin{array}{l}\text { Heart valve } \\
\text { replacement }\end{array}$ & 160,000 & 9000 & 10,000 & 12,500 & $6 \%$ & $6 \%$ & $8 \%$ \\
\hline Angioplasty & 57,000 & 11,000 & 13,000 & 13,000 & $19 \%$ & $23 \%$ & $23 \%$ \\
\hline Hip replacement & 43,000 & 9000 & 12,000 & 12,000 & $21 \%$ & $28 \%$ & $28 \%$ \\
\hline Hysterectomy & 20,000 & 3000 & 4500 & 6000 & $15 \%$ & $23 \%$ & $30 \%$ \\
\hline Knee replacement & 40,000 & 8500 & 10,000 & 13,000 & $21 \%$ & $25 \%$ & $33 \%$ \\
\hline Spinal fusion & 62,000 & 5500 & 7000 & 9000 & $9 \%$ & $11 \%$ & $15 \%$ \\
\hline
\end{tabular}

Source: ABILITY Magazine at http://www.abilitymagazine.com/pbb.html. 
tourist attractions, insurance, translation, etc., and its huge commercial potential and market profits are subject to national government and international Tourism agencies, medical institutions and policy makers attach great importance to turning medical tourism into a competitive new economic growth point [16]. Undoubtedly, the wave of globalization has brought new opportunities and challenges to the medical tourism industry. The "reverse globalization" of the source of tourists further highlights the importance of the decision-making process of cross-border medical tourists to the development of the industry. For the consumer purchase decision-making process, the research of foreign scholars started earlier. Suleyman Ozarslan systematically established a dynamic analysis framework for the consumer decision-making process, and proposed a 5-step decision-making process for demand, information collection, evaluation selection, decision purchase, and after-sales evaluation [17]. It is worth noting that although demand is the original driving force for consumers' desire to buy, price perception (such as cheap or expensive, reasonable or unreasonable) also plays an important role in the consumer's decision-making process. Some scholars pointed out that consumers' perception of price advantage helps to form purchasing preferences and brand loyalty, and is accustomed to measuring their purchase satisfaction by price standards [18].

It can be seen that the consumer purchase decision-making behavior is a dynamic process in which the consumer decides to purchase a specific product or service from mass-collecting information to objectively evaluate and select a product or service. The same is true for the decision-making process of cross-border medical tourists. It is a systematic and dynamic decision-making process, including the determination of requirements, the collection of information, the implementation of purchasing behavior and the evaluation of after-sales (see Figure 1). In the era of globalization, cross-border medical tourism is becoming the fastest-growing industry with the innovation of science and technology and the change of health concept. At the same time, the "reverse globalization" of passenger flow has intensified the competition in the countries of medical tourism. On the one hand, the rising cross-border medical tourism output value has made more countries rush into the medical tourism market, leading to increased market competition. On the other hand, the orderly competitive medical tourism market provides consumers with more choices. The flow is more diverse, and how to grasp the decision-making process of medical tourists to maximize the attraction of customers and seize market share has become the focus of policy makers.

In general, the decision-making process of cross-border medical tourism is a complex process. The first step is that patients' judge and identify their own medical needs based on their physical condition. Then, they collect information on treatment options from various sources for medical needs, and weigh the information collected to determine whether the cross-border medical tourism program is the treatment of choice for them. At this time, if it is decided to go to 


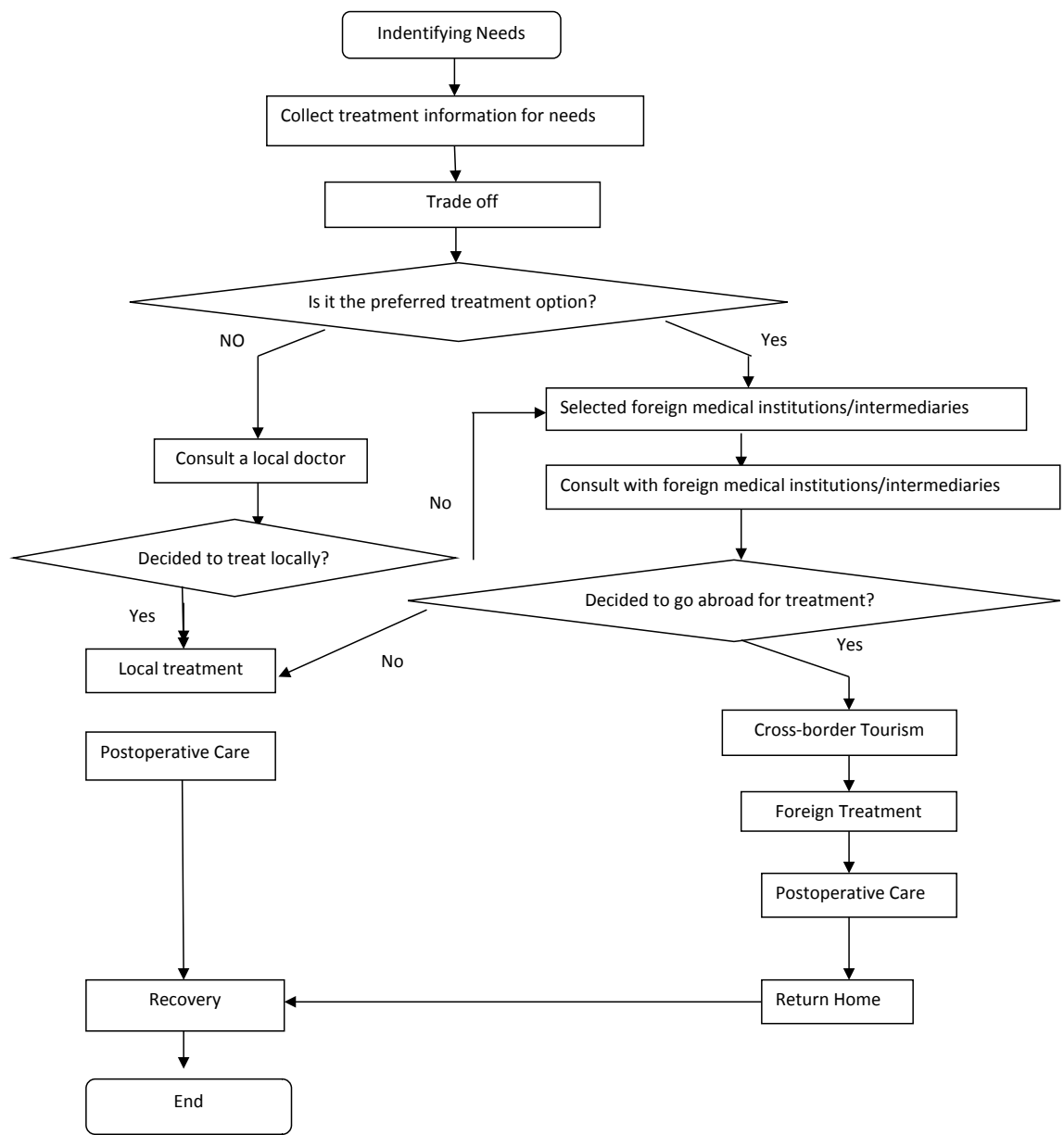

Figure 1. Decision-making flow chart for cross-border medical tourism.

a foreign medical institution for treatment, it will start to consult with foreign medical institutions in a targeted manner, and complete the process of cross-border medical care, post-operative care, returning to the home, and nursing at home according to the doctor's advice; If you decide to receive treatment at home under various trade-offs, consult a local doctor and complete a series of medical treatments under the guidance of a local doctor.

\subsection{Analysis of Cross-Border Medical Tourism Decision-Making Process}

Identifying needs is the first step in the process of medical tourists' sequential logic decision-making, and is an important driving force for the development of medical tourism industry. Scholars have conducted a lot of research on the motives of people's cross-border medical tourism, and found that identifying treatment needs is of great significance to the subsequent decision-making process. Ye (2011) found that a large number of medical tourists from mainland China have refused to adopt the policy of family planning to accept cross-border medical services in Hong Kong [19]. Moghimehfar and Nasr-Esfahani (2011) explained that the need to maintain religious identity makes Iran the preferred lo- 
cation for medical tourists in neighboring countries [20]. Accurate identification of medical needs by medical tourists can help to collect information in a targeted manner. The precise identification of the medical needs of consumers in medical tourism destinations helps to develop their own industrial strategies. Macao's demand for accurate and high-quality medical examination services for medical tourists in mainland China has become an emerging cross-border medical tourism destination by taking advantage of price, language and geographical location [21].

Once the needs are identified, information on the relevant treatment options needs to be collected for specific medical needs. Consumers weigh the pros and cons based on the information collected and determine the preferred treatment plan to achieve maximum satisfaction. According to the information collected, the medical tourism destination countries study the consumption preferences of medical tourists and take corresponding measures to increase the income of medical tourism in the country. Japanese consumers and Chinese consumers go to South Korea for medical services based on different medical needs. Chinese medical tourists focus on medical tourism project choices, prefer to receive plastic surgery in Korea, while Japanese consumers prefer rehabilitation and psychotherapy. The quality of service for medical tourism is highly valued [22]. Internet and friend recommendations are the main source of information for them. It is worth mentioning that the Japanese usually adopt the theoretical framework of planning behavior to plan their cross-border medical tourism. The long-term pressure from family, friends and work is the main reason for their travel to South Korea for psychotherapy [23].

Since the beginning of the 21 st century, the cross-border medical tourism industry has undergone tremendous changes, and the flow of tourists has shown a complex and ever-changing situation. It turned out that the wealthy class has developed into a large-scale industry for the pursuit of quality medical services, and ordinary medical tourists hope more involved in the decision-making process. Whether cross-border medical tourism is the first choice node in the decision-making process affects people's follow-up consultation and in-depth research. It is worth noting that it is first decided whether to go out and consult the corresponding medical institution in a targeted manner, or to consult different institutions before making a decision on whether or not to leave the country. The scholars have different views for the order of the two processes. Consultation with local medical institutions is likely to lead to decisions on receiving medical services in the local area. Consulting with foreign medical institutions or intermediaries can expand the range of people's choices, and finally decide to go abroad for medical services on the advice of foreign doctors [24]. The location of the consulting medical institution does not represent the destination of the final medical service, but the medical institution being consulted has a great influence on the decision-making of consumers and has certain advantages in giving priority to attracting customers. Therefore, the countries with 
medical tourism destinations should pay attention to the construction of medical consultation institutions in the development of cross-border medical tourism industry, and strive to attract customers in the information consultation stage.

After comprehensively weighing the pros and cons of all aspects, consumers make the final decision and go to the corresponding country or region to receive medical tourism services according to their respective choices. However, for treatment abroad, cross-border medical tourists may face challenges such as communication barriers and environmental adaptation. In the post-operative care stage, many medical institutions provide patients with recreational and recreational tourism and entertainment projects, so that patients can truly enjoy the value of medical and tourism coexistence. From the process of receiving medical services from the exit to the end of the cross-border medical tourism project, due to visas, medical institutions, hotels, catering, translation, insurance and many other departments, medical tourism destination countries should take effective measures to ensure the development of cross-border medical tourism. Undoubtedly, the development of cross-border medical tourism can bring huge benefits to the government's promotion of international medical service trade, integration of medical tourism industry chain and job creation. Among them, India has set up a special M-type visa for cross-border medical tourists (Medical Visa) [25].

\section{Discussion}

The convenience of cross-border medical tourism has promoted the development of globalization and the medical market. According to data from American Insurance Association [26], the trend of industrial internationalization has enabled medical insurers and non-medical insurers in the United States to continue to seek various medical treatments abroad, and that medical tourism is The world's fastest-growing industry, and the growth trend of medical tourism is not only reflected in the level of individual behavior, many institutions are exploring the potential commercial value of the industry. For example, in order to reduce costs, corporate employers use cross-border medical tourism as employee medical benefits and supplementary medical insurance, and US insurance companies also include cross-border medical tourism in insurance programs [27].

\subsection{Factors Affecting the Choice of Countries for Cross-Border Medical Tourism Destinations}

Figure 2 shows a two-stage conceptual model that influences the choice of countries and medical facilities for cross-border medical tourism. This model shows that no single factor is able to dominate the final decision, each of which plays an important role in cross-border medical tourism decision-making. Once people are conducting cross-border medical tourism, they must consider how to choose the right medical tourism destination and medical project facilities. The model in this paper is based on the two-stage model adopted by Palvia (2017) to 


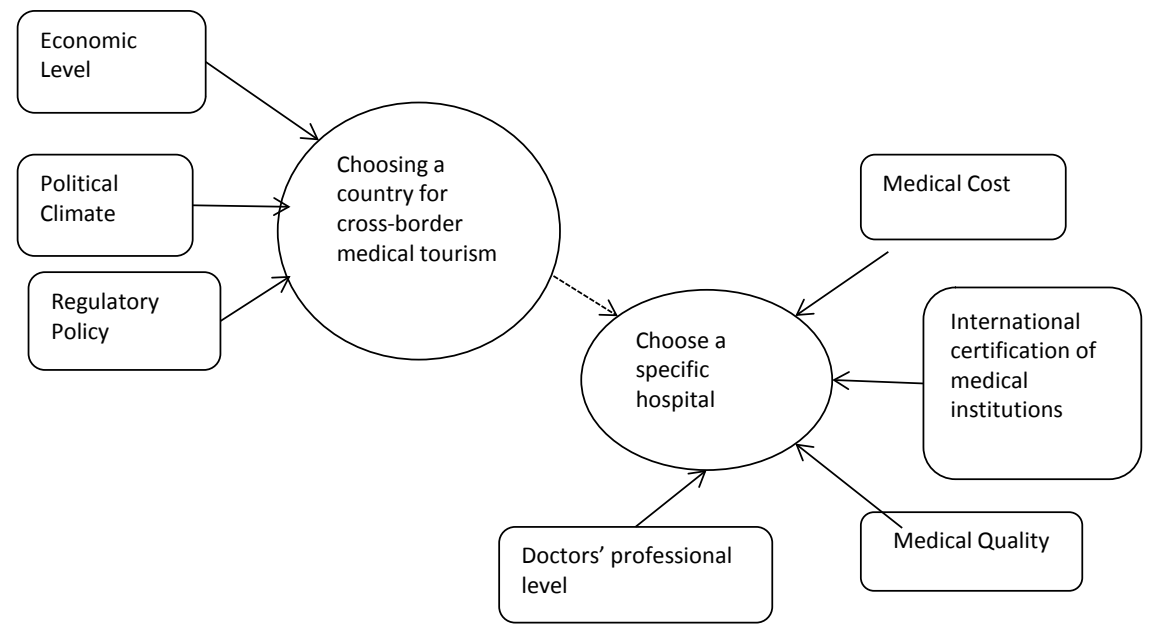

Figure 2. Factors affecting the choice of countries and medical facilities for cross-border medical tourism destinations.

analyze IT global procurement service selection suppliers. The first phase determines the country, the second phase determines the supplier, and the factors affecting the country's choice include the political system and communication infrastructure construction, management systems, labor quality and quantity, judicial and legal systems, language/culture, etc; the factors that influence suppliers include the matching of supplier capabilities and customer needs, cost considerations, supply speed, product quality, etc. [28].

\subsubsection{Economic Level}

The cross-border medical tourism industry can bring huge economic benefits to medical tourism destination countries and play a huge role in leading economic development, promoting social progress, and promoting technological innovation. The scale of cross-border medical tourists is huge, the integration of medical industry and international tourism industry, and the deepening of cross-border medical tourism industry exchange and cooperation have made the competitiveness and influence of the medical tourism industry continue to improve. The international image reflected by the economic level is a key factor for consumers to consider when choosing a medical tourism destination country. The driving force of the medical tourism destination country to develop the cross-border medical tourism industry is largely from the economic level. As a leader in the cross-border medical tourism market, India's development is also driven by economic considerations. In order to accelerate the development of the medical tourism industry and promote economic growth, the Indian government has adopted a series of measures such as tax cuts [29].

\subsubsection{Political Climate}

Political stability and long-lasting is the premise of national development. For a long time, security is the first consideration for cross-border medical tourists. The vigorous development of cross-border medical tourism industry requires a 
stable political climate. American medical tourists are more willing to go to countries with stable political and terrorist threats to obtain specific medical services. Thailand is a leader in medical tourism in Asia, but its unstable political situation puts pressure on the cross-border medical tourism industry. A stable political climate makes it easier to gain the trust of cross-border medical tourists, and trust is critical to countries that are committed to developing medical tourism [30]. Building trust with consumers is likely to generate consumer loyalty. The brand effect is used to drive the rapid development of the entire industry.

\subsubsection{Regulatory Policy}

Regulatory policies accurately positioning the country's strategy for developing medical tourism through planning or legislation is an important measure to enhance the cross-border medical tourism industry value chain. Due to the promulgation and implementation of the US Health Insurance Portability and Accountability Act (HIPPA, 1996), medical tourists from the United States will consider the country's regulatory policies more when choosing a destination for medical tourism. Part of the reason for cross-border medical tourism for many people is because their own regulatory policies have led to medical services such as abortions that are not allowed to go abroad for medical services. The privacy protection of specific medical items (such as cosmetic surgery and transgender) brought about by medical supervision policies are also important factors affecting people's choice of countries for medical tourism. The US health care system uses relevant regulatory policies and medical insurance regulations to protect patient rights, and most states require residents to purchase liability insurance and medical accident insurance [31].

\subsection{Factors Affecting the Choice of Cross-Border Medical Tourism Hospitals}

As a new industry with great development potential and involving many stakeholders, cross-border medical tourism attracts the attention of the government, medical institutions, social citizens and other subjects. Brand familiarity, access to medical services, medical costs, medical quality and legality of medical services are the main driving forces for people to go abroad for medical tourism services. Brand medical service institutions have become the first choice for overseas medical tourists [32]. Therefore, studying how patients choose cross-border medical tourism hospitals is conducive to improving hospital income and visibility.

\subsubsection{Medical Costs}

In fact, high quality and low price are one of the important characteristics of medical tourism. Therefore, medical tourism expenses are one of the main factors affecting the development of cross-border medical tourism. In recent years, due to the high medical costs of developed countries, many developing countries have rapidly emerged with the advantage of price, becoming a medical tourism 
country with great development potential. Increasing medical costs have become the leading cause of cross-border medical tourism in the United States. World Bank research shows that medical costs in the United States are significantly higher than in other countries, and the price advantage of the US medical tourism industry continues to decline [33]. In the same medical project, the cost of medical treatment in Thailand and India is 50\% - 90\% lower than that in the United States, which promotes the prevalence of cross-border medical tourism. Thailand, India, Malaysia and other countries have seized the opportunity of industrial internationalization and become an important medical tourism destination country with low cost of medical treatment. Due to the significant difference in the cost of medical tourism services between the United States and India, India has become one of the most popular destinations for medical tourism in the United States. Labor costs in developed countries are generally high, and according to data from the US Medical Care Medical Assistance Center, more than $70 \%$ of medical costs are labor-related. This shows that the labor cost advantage of developing countries has become a weapon for the development of the medical tourism industry.

\subsubsection{International Certification of Medical Institutions}

International certification is one of the most important criteria for tourists to judge the qualifications of foreign medical institutions. Foreign medical institutions have been committed to meeting or exceeding the quality standards of American medical care. Currently, international medical institutions' certification standards include The Australian Council on Healthcare Standards (ACHS), the NHS Regional Accreditation Scheme (TAS), and the Canadian Health Services Commission Standard (The Canadian Council on Health Services (CCHSA), The Joint Commission International (JCI). Among them, the national standard certification of the United States Medical Accreditation Joint Commission is most sought after by overseas medical institutions, and JCI certification of overseas medical institutions has become an essential element for attracting international medical tourists. JCI's international certification is based on a rigorous evaluation of the criteria and the review process for a comprehensive review of overseas medical institutions. If the results of the review meet all the accreditation requirements, the overseas medical institutions will be eligible for accreditation, and then the medical institutions will be required to develop strategies. Sexual improvement plan, clear improvement strategy and method, and the validity of one certification result is only 3 years. After 3 years, it is decided whether to apply for accreditation again. In terms of JCI certification, Thailand is at the forefront of many developing countries. Bumrungrad International Hospital in Bangkok, Thailand is a pioneer in international medical services and the first (2002) JCI-certified hospital in Asia [34]. In addition, India, China, Singapore, Brazil, the United Arab Emirates and other countries or regions are actively applying for JCI international certification and become a prominent representative of JCI international certification. Most overseas medi- 
cal institutions began to apply for JCI's international certification qualification in 2006. Whether or not to obtain JCI's international certification has become an important factor affecting the choice of medical institutions for overseas medical tourists, making the medical tourism industry appear to break through the national trend towards internationalization.

\subsubsection{Medical Quality}

Although obtaining JCI international certification can guarantee the medical quality level of medical institutions to a certain extent, having JCI certification qualification does not mean that people can completely ignore the quality of medical care when choosing overseas medical institutions. High quality and low price is an important bargaining chip for developing countries to attract overseas medical tourists. However, many medical institutions over-promoting low-priced selling points may cause questions from overseas medical tourists: Can such low prices guarantee high-quality medical services? The price advantage does not determine people's final choice. People go abroad to seek medical tourism services in order to enjoy the best quality medical services within an acceptable price range.

\subsubsection{Professional Level of the Doctor}

The professional level of doctors is the core element of the entire medical tourism service project. Overseas medical institutions often put the professional level of doctors at an important position when promoting their own advantages. Whether to receive professional training and obtain medical certificates in developed countries such as the United States has become a common standard for evaluating the professional level of doctors. The famous Bumrungrad International Hospital in Thailand announced in 2005 that more than 200 doctors in the hospital have overseas training experience and training certificates. The doctor's language ability is also an important factor affecting patients' decision-making. The doctor's English communication ability provides barrier-free language communication guarantee for cross-border medical patient consultation and follow-up care services. India has always been one of the most popular medical tourism destinations for American patients. In addition to low prices and excellent services, Indian doctors' English communication skills contribute to the development of the medical tourism industry [35].

\section{Conclusions}

Medical tourism is a new type of industry formed by the intersection and integration of the industry. The great development prospects of the medical tourism industry and its promotion of economic development have attracted much attention. This paper focuses on the decision-making model of cross-border medical tourism. Establishing a two-stage decision-making model to analyze the factors that patients should consider when selecting cross-border medical tourism services countries and medical institutions. As a combination of medical and 
tourism, the medical tourism industry has unique development advantages. The "reverse globalization" of customer flow has experienced the development of cross-border medical tourism. The decision-making model for cross-border medical tourism is also more important.

According to the development of cross-border medical tourism and the above-mentioned medical tourism decision-making model, China has great potential to develop cross-border medical tourism industry. It needs to clarify its own development advantages and current competitive situation, and explore the cross-border medical tourism source. The driving factors of mobility, at the same time, provide guidance and support at the policy level, improve industrial supervision policies, and clarify the strategic positioning of the industry. Specifically, China needs to pay attention to the development of cross-border medical tourism industry:

Firstly, identify self-development advantages and current competitive situation. China has a vast territory with rich medical tourism resources, from natural landscapes to historical sites, with the potential and advantages of developing cross-border medical tourism. Vigorously developing China's Chinese medical culture and developing China's cross-border medical tourism brand competitiveness will help develop the development of medical tourism, spread Chinese medicine culture, and add momentum to the development of China's medical tourism industry.

Secondly, understand the key factors affecting the development of cross-border medical tourism. Under the background of industrial internationalization, grasping the driving factors of customer flow will undoubtedly bring unlimited business opportunities to the development of medical tourism industry. The driving factor for the flow of cross-border medical tourism tourists is to break through the development of the medical tourism industry. To divide the market share in a highly competitive market, the state and medical institutions need to formulate specific strategies and countermeasures for each driver. The formation of brand loyalty plays a key role in consumer decision-making and the growth of industry output. Therefore, if China can develop its own cross-border medical tourism brand service project and carry out more professional medical tourism business, it will quickly improve China's international reputation of cross-border medical tourism.

Finally, improve regulatory policies and give policy support. The privacy protection and scientific ethics and legality disputes of medical tourism projects are the focus of a country's regulatory policy. At the same time, when the medical needs and interests of local residents and foreign medical tourists conflict, how to balance the relationship between fairness and interests is also the key issues. Political developers need to consider when improving their regulatory policies. Under the premise of limited medical resources, the development of cross-border medical tourism industry can not only focus on profit-seeking, but also to ensure fairness, avoid investment concentrated in the field of medical tourism, and make public medical investment insufficient, and high-quality medical person- 
nel flow to private medical institutions.

\section{Conflicts of Interest}

The author declares no conflicts of interest regarding the publication of this paper.

\section{References}

[1] Han, H.S. and Jinsoo, H. (2013) Multi-Dimensions of the Perceived Benefits in a Medical Hotel and Their Roles in International Travelers' Decision-Making Process. International Journal of Hospitality Management, 35, 100-108. https://doi.org/10.1016/j.ijhm.2013.05.011

[2] Iordache, C. and Ciochina, I. (2014) Medical Tourism Industry Challenges in the Context of Globalization. Management Strategies Journal, 7, 62-70.

[3] Bell, D., Holliday, R., Ormond, M. and Mainil, T. (2015) Transnational Healthcare, Cross-Border Perspectives. Social Science \& Medicine, 124, 284-289. https://doi.org/10.1016/j.socscimed.2014.11.014

[4] Picazo, O.F. (2013) Medical Tourism in the Philippines: Market Profile, Benchmarking Exercise, and s.w.o.t. Analysis. Working Papers.

[5] Hanefeld, J., Smith, R., Horsfall, D. and Lunt, N. (2014) What Do We Know about Medical Tourism? A Review of the Literature with Discussion of Its Implications for the UK National Health Service as an Example of a Public Health Care System. Journal of Travel Medicine, 21, 410-417. https://doi.org/10.1111/jtm.12147

[6] Connell, J. (2013) Contemporary Medical Tourism: Conceptualisation, Culture and Commodification. Tourism Management, 34, 1-13.

https://doi.org/10.1016/j.tourman.2012.05.009

[7] Smith, R., Martínez, Á.M. and Chanda, R. (2011) Medical Tourism: A Review of the Literature and Analysis of a Role for Bi-Lateral Trade. Health Policy, 103, 276-282. https://doi.org/10.1016/j.healthpol.2011.06.009

[8] Tattara, G. (2010) Medical Tourism and Domestic Population Health. Social Science Electronic Publishing.

[9] Beladi, H., Chao, C.C., Ee, M.S. and Hollas, D. (2015) Medical Tourism and Health Worker Migration in Developing Countries. Economic Modelling, 46, 391-396. https://doi.org/10.1016/j.econmod.2014.12.045

[10] Patrichi, I.C., and Dodu, S.P. (2014) Medical Tourism-A Business or a Necessity? Revista De TurismStudii Si Cercetari in Turism, 18.

[11] Bookman, M.Z. and Bookman, K.R. (2007) Medical Tourism in Developing Countries. Palgrave Macmillan. https://doi.org/10.1057/9780230605657

[12] Horowitz, M.D., Rosensweig, J.A. and Jones, C.A. (2007) Medical Tourism: Globalization of the Healthcare Marketplace. Medgenmed Medscape General Medicine, 9 , 33.

[13] Connell, J. (2006) Medical Tourism: Sea, Sun, Sand and Surgery. Tourism Management, 27, 1093-1100. https://doi.org/10.1016/j.tourman.2005.11.005

[14] Alberti, F. and Pizzurno, E. (2013) Competitiveness Policies for Medical Tourism Clusters: Government Initiative in Thailand. Portuguese Journal of Nephrology \&amp; Hypertension.

[15] Debata, B.R., Patnaik, B., Mahapatra, S.S. and Sreekumar, S. (2013) Efficiency Measurement amongst Medical Tourism Service Providers in India. International Jour- 
nal for Responsible Tourism, 1, 24-31.

[16] Underwood, H.R. and Makadon, H.J. (2010) Medical Tourism: Game-Changing Innovation or Passing Fad? Healthcare Financial Management, 64, 112-114.

[17] Ozarslan, S. and Eren, P.E. (2018) MobileCDP: A Mobile Framework for the Consumer Decision Process. Information Systems Frontiers, 2, 1-22. https://doi.org/10.1007/s10796-015-9601-2

[18] Ryu, K.S. and Han, H.S. (2010) Influence of the Quality of Food, Service, and Physical Environment on Customer Satisfaction and Behavioral Intention in Quick-Casual Restaurants: Moderating Role of Perceived Price. Journal of Hospitality \& Tourism Research, 34, 310-329. https://doi.org/10.1177/1096348009350624

[19] Ye, B.H., Qiu, H.Z. and Yuen, P.P. (2011) Motivations and Experiences of Mainland Chinese Medical Tourists in Hong Kong. Tourism Management, 32, 1125-1127. https://doi.org/10.1016/j.tourman.2010.09.018

[20] Moghimehfar, F. and Nasresfahani, M.H. (2011) Decisive Factors in Medical Tourism Destination Choice: A Case Study of Isfahan, Iran and Fertility Treatments. Tourism Management, 32, 1431-1434. https://doi.org/10.1016/j.tourman.2011.01.005

[21] Chingchi, L., Cros, H.D., Tzengai, V. and Hall, C.M. (2011) Macao's Potential for Developing Regional Chinese Medical Tourism. Tourism Review, 66, 68-82. https://doi.org/10.1108/16605371111127242

[22] Yu, J.Y. and Taegyou, K. (2015) A Cross-Cultural Study of Perceptions of Medical Tourism among Chinese, Japanese and Korean Tourists in Korea. International Journal of Nursing Education Scholarship, 33, 80-88.

[23] Misung, L., Han, H.S. and Lockyer, T. (2012) Medical Tourism-Attracting Japanese Tourists for Medical Tourism Experience. Journal of Travel \& Tourism Marketing, 29, 69-86. https://doi.org/10.1080/10548408.2012.638564

[24] Hibbard, J.H., Stockard, J., Mahoney, E.R. and Tusler, M. (2010) Development of the Patient Activation Measure (pam): Conceptualizing and Measuring Activation in Patients and Consumers. Health Services Research, 39, 1005-1026.

[25] Chinai, R. and Goswami, R. (2007) Medical Visas Mark Growth of Indian Medical Tourism. Bulletin of the World Health Organization, 85, 164. https://doi.org/10.2471/BLT.07.010307

[26] Cortez, N. (2008) Patients without Borders: The Emerging Global Market for Patients and the Evolution of Modern Health Care. Social Science Electronic Publishing, 83, 71-132.

[27] Marlowe, J., Sullivan, P., Marlowe, J., Sullivan, P., Marlowe, J., Sullivan, P., et al. (2007) Medical Tourism: The Ultimate Outsourcing. Human Resource Planning.

[28] Palvia, S.C.J. (2017) Global Sourcing of Services: A Two Stage Model for Selecting a Vendor: Strategies, Issues and Challenges. World Scientific Book Chapters.

[29] Rikke, S. and Despena, A. (2015) American Medical Tourism in India: A Retrospective Health Policy Analysis. International Journal for Responsible Tourism, 4.

[30] Han, H.S. and Hyun, S. (2013) Image Congruence and Relationship Quality in Predicting Switching Intention: Conspicuousness of Product Use as a Moderator Variable. Journal of Hospitality \& Tourism Research, 37, 303-329. https://doi.org/10.1177/1096348012436381

[31] Mello, M.M. (2006) Understanding Medical Malpractice Insurance: A Primer. Synthesis Project Research Synthesis Report, 22, 269.

[32] Glinos, I.A., Baeten, R. and Boffin, N. (2006) Cross-Border Contracted Care in Bel- 
gian Hospitals. Patient Mobility in the European Union.

[33] Mattoo, A. and Rathindran, R. (2005) Does Health Insurance Impede Trade in Health Care Services? Social Science Electronic Publishing, No. 3, 468. https://doi.org/10.1596/1813-9450-3667

[34] Wilson, A. (2011) Foreign Bodies and National Scales: Medical Tourism in Thailand. Body \& Society, 17, 121-137. https://doi.org/10.1177/1357034X11400923

[35] Hazarika, I. (2010) Medical Tourism: Its Potential Impact on the Health Workforce and Health Systems in India. Health Policy \& Planning, 25, 248.

https://doi.org/10.1093/heapol/czp050 